Review Article

\title{
Processing and Compatibility of Corydalis yanhusuo: Phytochemistry, Pharmacology, Pharmacokinetics, and Safety
}

\author{
Lingyan Wu $\mathbb{D}^{1,2}$ Yang Yang $\left(\mathbb{D},{ }^{2}\right.$ Zhujun Mao $\mathbb{D}^{2},{ }^{2}$ Jianjun $W u\left(\mathbb{D},{ }^{2}\right.$ Dan Ren $\mathbb{D},,^{1,2}$ Bo Zhu $\left(\mathbb{D},{ }^{2}\right.$ \\ and Luping Qin (iD) ${ }^{2}$
}

${ }^{1}$ College of Pharmacy, Shaanxi University of Chinese Medicine, Xianyang 712046, China

${ }^{2}$ School of Pharmaceutical Sciences, Zhejiang Chinese Medical University, Hangzhou 310053, China

Correspondence should be addressed to Bo Zhu; zhubo@zcmu.edu.cn and Luping Qin; lpqin@zcmu.edu.cn

Received 2 July 2021; Accepted 22 November 2021; Published 30 December 2021

Academic Editor: Dan Su

Copyright (C) 2021 Lingyan Wu et al. This is an open access article distributed under the Creative Commons Attribution License, which permits unrestricted use, distribution, and reproduction in any medium, provided the original work is properly cited.

Processed and polyherbal formulations (compatibility) are widely used in traditional Chinese medicine (TCM). However, processing and compatibility may alter the efficacy and safety of herbal medicines, and therefore, evaluating the herbal medicines changes after processing and compatibility is important for their safety. Since Corydalis yanhusuo (Y.H.Chou \& Chun C.Hsu) W.T.Wang ex Z.Y.Su \& C.Y.Wu (Family: Papaveraceae and Genera: Corydalis), a traditional medicinal plant in China, Japan, Korea, and other Asian countries, has been used for treating a wide range of medical conditions, it is an ideal representative of studying the effects of processing and compatibility on efficacy and toxicity. In this paper, information was obtained by searching electronic databases, classic books, $\mathrm{PhD}$ and MSc dissertations, local conference papers, and unpublished materials prior to July 2021. We provide a summary of the phytochemistry, pharmacology, pharmacokinetics, quality control, and safety of $C$. yanhusuo under various processing or compatibility conditions. Based on our findings, vinegar processing is probably the best $C$. yanhusuo processing method, which could increase the absorption rate of tetrahydropalmatine (THP) in the heart, liver, spleen, lung, and brain tissues and alleviate mice muscle tremors and liver damage caused by C. yanhusuo. These results indicate that processing and compatibility can reduce toxicity and increase the efficacy of C. yanhusuo. The information provides an expanded understanding of the efficacy and toxicity mechanisms of TCM compounds, which is valuable for industrial production quality control and future drug research.

\section{Introduction}

Prior to incorporation as pharmaceutical ingredients and use as medicinal decoctions in clinics, medicinal plants are processed through a series of traditional Chinese techniques such as washing, cutting, and frying with various excipients [1]. Processing, an essential step for herbal medicinal preparations, is a pharmaceutical process applied for different therapeutic purposes, which can increase efficacy and also reduce toxicity of herbal medicines [2]. Meanwhile, compatibility (polyherbal formulations), which entails combining multiple formulae of traditional Chinese medicine (TCM) (two or more medicines) selectively according to the characteristics of the individual medical plants, is a common practice in TCM aimed at achieving balanced or synergistic effects [3]. Therefore, evaluating the herbal medicines changes after processing and compatibility is important for their safety.

Corydalis yanhusuo (Y.H.Chou \& Chun C.Hsu) W.T.Wang ex Z.Y.Su \& C.Y.Wu (Synonym: Corydalis turtschaninovii f. yanhusuo Y.H.Chou \& Chun C.Hsu; https://www.theplantlist.org), a perennial herb with a 300year cultivation history, is a typical medicinal raw material that goes through processing and compatibility to achieve a better therapeutic effect (see Figure 1). Its rhizome, known as "Yuanhu," is often used to treat miscellaneous medical disorders such as pain, insomnia, cardiovascular diseases, hypertension, gastric ulcers, cancer, and inflammation [4]. Additionally, Corydalis yanhusuo W.T.Wang (C. yanhusuo) is often combined with other medicinal plants such as 


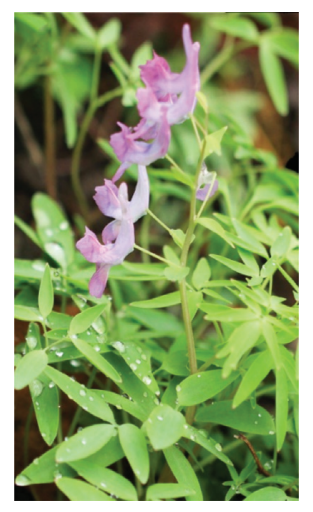

(a)
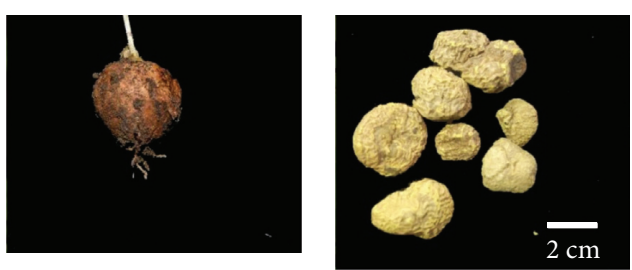

(b)

(c)

Figure 1: (a) Above ground part of C. yanhusuo, (b) tuber of C. yanhusuo, and (c) commercial tubers of C. yanhusuo.

Angelica dahurica in classical TCM prescriptions for enhanced analgesic activity. However, when used in excess (more than $3 \mathrm{~g} /$ day), minor toxicity including liver toxicity and muscle tremor in rats have been reported [5]. According to the Chinese Pharmacopoeia 2020 edition, the "standard" processing method is stir-frying C. yanhusuo with vinegar. In this process, C. yanhusuo and vinegar are combined at a ratio of $5: 1$ and heated at a low temperature until the vinegar has seeped into the medicinal material [6]. Other methods include "stir-frying" with salt, rice wine, clam powder, and glutinous rice, which have been reported in the ancient medical books and local practices, including "Leigong Paozhi Lun” (《雷公炮制论》, Northern-Southern Dynasties, A.D. 588) and "Compendium of Materia Medica" (《本 草纲目》, Ming Dynasty, A.D. 1578) [7]. The main bioactive ingredients of C. yanhusuo are alkaloids, including tetrahydropalmatine, tetrahydrocoptisine, corydaline, tetrahydroberberine, palmatine, coptisine, and dehydrocorydaline. However, possible interactions and the mechanisms of action of the bioactive compounds present in C. yanhusuo are so far unclear. Typical formulation compositions and therapeutic effects of the C. yanhusuo are summarized in Supplementary Table S1 (https://www.yaozh.com, last accessed on July 1, 2021).

C. yanhusuo processed products and compatible preparations are currently being used in clinical settings; therefore, C. yanhusuo is an ideal representative of studying the effects of processing and compatibility on efficacy and toxicity. Changes in pharmacokinetics, which can also explain the changes in drug efficacy, serve as a knowledge base for C. yanhusuo processing and compatibility. Processing or compatibility may result in changes in the chemical composition, pharmacological activity, or safety of C. yanhusuo and subsequent effects on its application in TCM. In this paper, we provide useful information on the phytochemistry, pharmacological activity, pharmacokinetics, and toxicity of C. yanhusuo under various processing and compatibility methods, based on recent studies. This information provides an expanded understanding of the efficacy and toxicity mechanisms of C. yanhusuo, which is valuable for industrial production quality control and future drug research, and provides insight into safe and rational use of processed and polyherbal formulations of C. yanhusuo.

\section{Materials and Methods}

Information on C. yanhusuo was obtained by searching electronic databases, including Web of Science, ScienceDirect, BaiduScholar, PubMed, SciFinder, CNKI, and GoogleScholar, as well as classic books of Chinese herbal medicine, Pharmacopoeia, $\mathrm{PhD}$ and MSc dissertations, local conference papers, and unpublished materials prior to July 2021. The keywords were Corydalis yanhusuo, processing, compatibility, phytochemistry, pharmacology, pharmacokinetics, safety, and other related words with no time limit (all fields). Due to the lack of data on quality control, the results of several articles were initially considered controversial; subsequently, these articles were discarded because they were scientifically unreliable. In this paper, a total of 41 documents were embraced.

\section{Phytochemistry}

Previous phytochemistry studies have revealed that C. yanhusuo has more than 100 compounds, including fatty acids, alkaloids, volatile oils, saccharides, and other compounds [4]. Fatty acids and alkaloids, as dominant compounds in C. yanhusuo, showed obvious content change after processing and compatibility.

Fatty acids in C. yanhusuo possess anti-inflammatory and antitumor activities [8]. A GC-MS study showed that 7 fatty acids, 2 olefins, and 2 lipids components were identified in raw C. yanhusuo and vinegar C. yanhusuo at different concentrations. Among the 4 fatty acids with less than 20 carbon atoms (linolelaidic acid methyl ester; methyl oleate; methyl palmitate; methyl stearate) were more abundant in raw C. yanhusuo, while 3 fatty acids with more than 20 carbon atoms (methyl arachidate; methyl behenate; methyl lignocerate) were more abundant in vinegar C. yanhusuo. Unlike in raw C. yanhusuo, ethyl 4, 5, 6, 7-tetrahydro-3-methyl-2-formate indole could not be detected in vinegar processed C. yanhusuo. Therefore, further studies are needed to establish whether 4, 5, 6, 7tetrahydro-3-methyl-2-carboxylic acid ethyl indole can be used as an indicator for the presence of raw C. yanhusuo and vinegar C. yanhusuo [9]. 
Alkaloids are the main compounds of C. yanhusuo and have been confirmed to have significant analgesic, anti-inflammatory, and antitumor activities $[10,11]$. Their typical structures are shown in Figure 2. The auxiliary vinegar used in processing can be combined with C. yanhusuo alkaloids to form salt and increase their solubility, and the dissolution rate of alkaloids is improved; therefore, vinegar processing has been shown to result in increased amounts of protopine and tetrahydrocoptisine [12]. On the other hand, the amounts of berberine hydrochloride, palmatine hydrochloride, and dehydrocorydaline are significantly decreased during vinegar processing; this may be due to the decomposition of quaternary amine alkaloids in C. yanhusuo after vinegar boiling $[12,13]$. The influence of processing methods on the content of C. yanhusuo is compounded as shown in Figure 3 and Table 1. However, related research on some C. yanhusuo compounds is still at the initial stages. For instance, while volatile oils and polysaccharides are major active compounds in C. yanhusuo and possess anti-inflammatory, antibacterial, antitumor, and cerebral ischemia protective effects, so far, there are no studies on their possible changes before and after processing. Therefore, in future studies, it is necessary to evaluate the content, pharmacology, and safety of these components in processed C. yanhusuo. Based on the benefits offered by vinegar processing (e.g., increasing the content of effective ingredients in C. yanhusuo) and its long history in TCM, we believe that it is the most suitable method for processing C. yanhusuo $[13,14]$.

\section{Pharmacological Activities}

Owing to its wide range of pharmacological activities, C. yanhusuo has been used as a tonic for treating pain [15], insomnia [16], drug addiction [17], and hypertension [18]. In TCM, processed C. yanhusuo is believed to have superior pharmacological efficacy compared with raw C. yanhusuo. Rat (NIH species, weight 18-22 g, female) was orally administered with vinegar C. yanhusuo extracts $(10 \mathrm{~g} / \mathrm{kg})$ for 6 consecutive hours to test analgesic effect. The results showed that there was significant reduction in the number of writhing reactions (from $10.3 \pm 3.9$ times $/ 15$ mins to $7.8 \pm 2.8$ times $/ 15$ mins) and raise in the pain threshold (from $15.4 \pm 4.6 \mathrm{~s}$ to $43.7 \pm 5.2 \mathrm{~s}$ ) compared with raw C. yanhusuo $(10 \mathrm{~g} / \mathrm{kg})$ and positive drug indomethacin $(1 \mathrm{mg} / \mathrm{kg})$, indicating that vinegar C. yanhusuo has better analgesic effects $(P<0.05)$ [19]. However, the reliability of the study is limited by the lack of information on the content changes of C. yanhusuo constituents responsible for analgesia or an explanation of the analgesia mechanism. Rat (NIH species, weight 18-22 g, half male and female) was orally administered with vinegar C. yanhusuo extracts ( $4 \mathrm{~g} / \mathrm{kg}$ ) for 3 consecutive days to test anti-inflammatory effect. The results showed that there was significant reduction in ear swelling rate (from $8.8 \pm 2.7$ to $4.1 \pm 1.2$ ), as compared to the raw C. yanhusuo $(4 \mathrm{~g} / \mathrm{kg})$, suggesting that vinegar C. yanhusuo has better anti-inflammatory effect [19]. Yuanhu Zhitong prescription (consists of C. yanhusuo and Angelicae dahuricae; YHZT) was reported to have better analgesic effect compared with C. yanhusuo used individually [20].
In addition, polyherbal C. yanhusuo with other TCM, such as Curcuma longa and Melia toosendan, has enhanced the antitumor and antigastric ulcer effects as compared to single C. yanhusuo. Rat (SD species, weight 180-220 g, male) was orally administered with Jinlingzi powder extracts $(0.13 \mathrm{~g} / \mathrm{mL}$ ) (a combination of C. yanhusuo and Melia toosendan) for 12 consecutive days to test treatment effects on gastric ulcer. The dose volume was $1 \mathrm{~mL} / 100 \mathrm{~g}$ according to the weight of the rats. The results showed that there was significant reduction in $\mathrm{N}$-terminal brain natriuretic peptide precursor (NT) and increased interleukin-8 (IL-8), tumor necrosis factor- $\alpha$ (TNF- $\alpha)$, platelet-activating factor (PAF), thromboxane B2 (TXB2), and vascular endothelial growth factor (VEGF) in serum, neutral neutrophil activation, which was as effective as the positive drug omeprazole $(0.36 \mathrm{mg} / \mathrm{mL})$. The results were statistically significant $(P<0.05)$, indicating that the Jinlingzi powder extracts had a significant effect on the treatment of the ulcer [21]. This is the first time to elucidate the rationality of the prescription in view of pharmacokinetic and pharmacological effects. In addition, YanhusuoSan (a combination of C. yanhusuo and Curcuma longa) extract-containing medium $(100 \mu \mathrm{L})$ treatment on MDA-MB-231 cells for $48 \mathrm{~h}$ reduced the cell invasion ability, suppressed the level of phospho-extracellular regulated protein kinases ( $\mathrm{p}$-ERK), and exhibited the strongest anticancer cell proliferation effect at the ratio of 3 : 2 , which provided a plausible molecular basis of antitumor effect of C. yanhusuo and Curcuma longa [22].

In the future, reasonable quality content standards for nonalkaloids such as volatile oils, organic acids, and polysaccharides are essential, which can help establish a credible theoretical and experimental foundation for C. yanhusuo research.

\section{Pharmacokinetics Studies}

5.1. Absorption. Changes in efficacy and toxicity of processed and compatible C. yanhusuo can also be explicated by the altered pharmacokinetics properties. So far, pharmacokinetics research has mainly focused on C. yanhusuo alkaloids. Additionally, beagle $\operatorname{dog}(12 \pm 2 \mathrm{~kg}$, male) was orally administered with YHZT extracts $(0.15 \mathrm{~g} / \mathrm{kg})$ to detect pharmacokinetics parameters. The results showed that there was significant $(P<0.001)$ raise in the $\mathrm{Cmax}, t_{1 / 2}, \mathrm{AUC}_{0-\infty}$, and $\mathrm{AUC}_{0-t}$ of dehydrocorydaline, tetrahydropalmatine, protopine, palmatine, columbamine, and berberine compared to raw C. yanhusuo $(0.0486 \mathrm{~g} / \mathrm{kg})$, and the $t_{\max }$ of the two groups was shorter than $1 \mathrm{~h}$. The above results suggest that vinegar processing and compatibility have longer elimination time and better bioavailability [23]. Moreover, the average plasma concentration curves of these compounds showed multipeak phenomenon, which may be related to distribution, reabsorption, and enterohepatic circulation [24]. Additionally, the polyherb of C. yanhusuo and Angelicae dahuricae is proved to increase the under curve area $\left(\mathrm{AUC}_{0-t}\right), \mathrm{AUC}_{0-\infty}$, and $C_{\max }$ of CDL and THP, as well as delay mean residence time $\left(\mathrm{MRT}_{0-T}\right.$ and $\left.\mathrm{MRT}_{0-\infty}\right)$ of CDL, suggesting a synergistic effect between alkaloid and coumarin, which is related to their increased absorption 

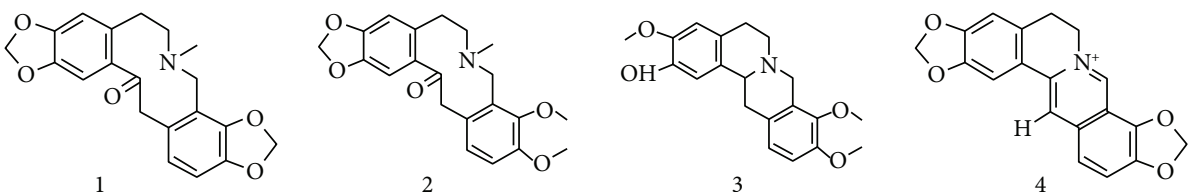

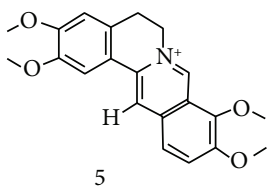<smiles>COC1=CC=C2C[N+]1=CC1=C2CCc2cc3c(cc21)OCO3</smiles><smiles>COc1cc2c(cc1OC)-c1c(C)c3ccc(OC)c(OC)c3c[n+]1CC2</smiles><smiles>COc1cc2c(cc1OC)C1Cc3ccc(OC)c(OC)c3CN1CC2</smiles><smiles>COc1ccc2c(c1OC)CN1CCc3cc4c(cc3C1C2)OCO4</smiles><smiles>COc1cc2c(cc1OC)C1c3cc(OC)c(OC)c(c3CN3CCC2c2ccc(OC)c(OC)c2C3)C1C</smiles>

10

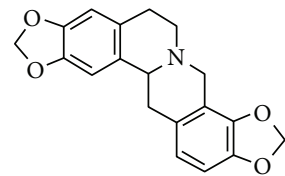

11

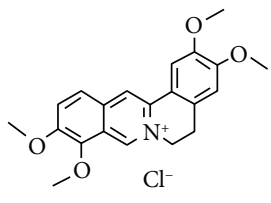<smiles></smiles><smiles></smiles>

12

14

FIGURE 2: Alkaloids isolated from C. yanhusuo.

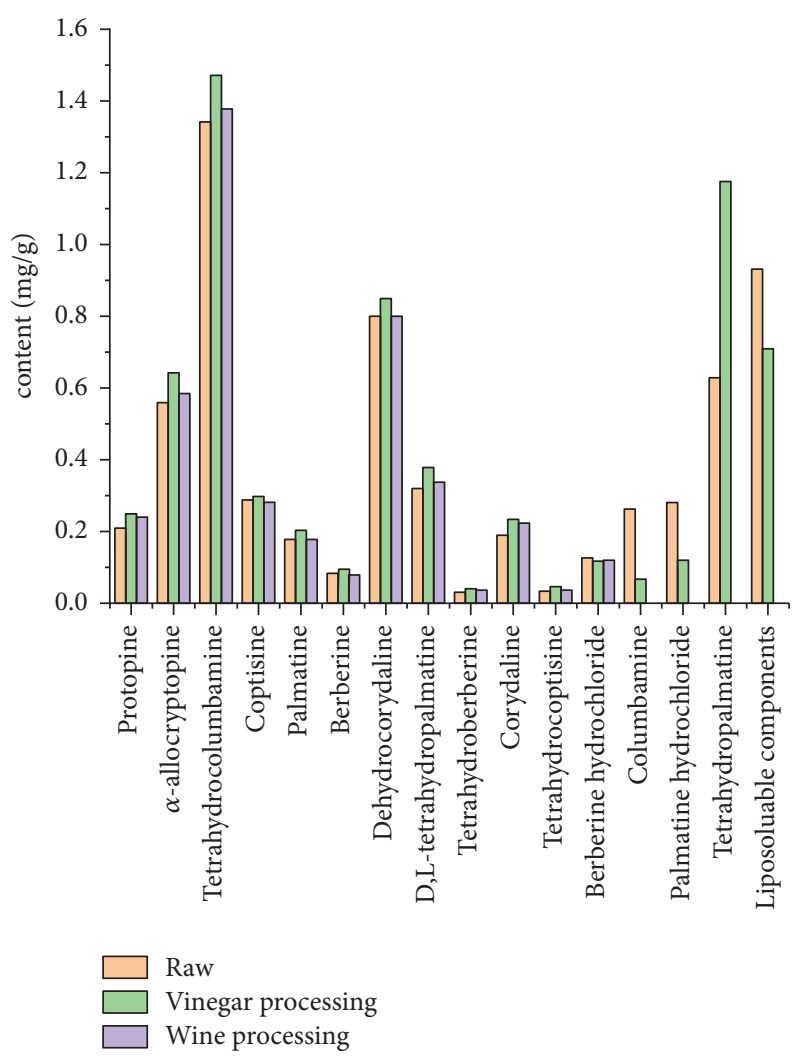

FIGURE 3: Quantitative changes on the content of C. yanhusuo compounds under different processing methods. 
TABLE 1: Quantitative changes on the content of Corydalis yanhusuo compounds under different processing methods.

\begin{tabular}{|c|c|c|c|c|}
\hline Number & Compounds & Processing method & Processing content (mg/g) & Reference \\
\hline & & None & 0.216 & \\
\hline \multirow[t]{3}{*}{1} & Protopine & Stir-baking with vinegar & $0.249 \uparrow$ & [12] \\
\hline & & Stir-baking with rice wine & $0.241 \uparrow$ & \\
\hline & & None & 0.559 & \\
\hline \multirow[t]{3}{*}{2} & $\alpha$-allocryptopine & Stir-baking with vinegar & $0.644 \uparrow$ & {$[12]$} \\
\hline & & Stir-baking with rice wine & $0.585 \uparrow$ & \\
\hline & & None & 1.340 & \\
\hline \multirow[t]{3}{*}{3} & Tetrahydrocolumbamine & Stir-baking with vinegar & $1.479 \uparrow$ & {$[12]$} \\
\hline & & Stir-baking with rice wine & $1.379 \uparrow$ & \\
\hline & & None & 0.290 & \\
\hline \multirow[t]{3}{*}{4} & Coptisine & Stir-baking with vinegar & $0.300 \uparrow$ & {$[12]$} \\
\hline & & Stir-baking with rice wine & $0.281 \downarrow$ & \\
\hline & & None & 0.180 & \\
\hline \multirow[t]{3}{*}{5} & Palmatine & Stir-baking with vinegar & $0.177 \downarrow$ & {$[12]$} \\
\hline & & Stir-baking with rice wine & $0.204 \uparrow$ & \\
\hline & & None & 0.084 & \\
\hline \multirow[t]{3}{*}{6} & Berberine & Stir-baking with vinegar & $0.078 \downarrow$ & {$[12]$} \\
\hline & & Stir-baking with rice wine & $0.092 \uparrow$ & \\
\hline & & None & 0.800 & \\
\hline \multirow[t]{3}{*}{7} & Dehydrocorydaline & Stir-baking with vinegar & $0.797 \downarrow$ & {$[12]$} \\
\hline & & Stir-baking with rice wine & $0.851 \uparrow$ & \\
\hline & & None & 0.321 & \\
\hline \multirow[t]{3}{*}{8} & D, L-tetrahydropalmatine & Stir-baking with vinegar & $0.339 \uparrow$ & {$[12]$} \\
\hline & & Stir-baking with rice wine & $0.381 \uparrow$ & \\
\hline & & None & 0.032 & \\
\hline \multirow[t]{3}{*}{9} & Tetrahydroberberine & Stir-baking with vinegar & $0.040 \uparrow$ & {$[12]$} \\
\hline & & Stir-baking with rice wine & $0.036 \uparrow$ & \\
\hline & & None & 0.189 & \\
\hline \multirow[t]{3}{*}{10} & Corydaline & Stir-baking with vinegar & $0.224 \uparrow$ & {$[12]$} \\
\hline & & Stir-baking with rice wine & $0.233 \uparrow$ & \\
\hline & & None & 0.033 & \\
\hline \multirow[t]{3}{*}{11} & Tetrahydrocoptisine & Stir-baking with vinegar & $0.036 \uparrow$ & {$[12]$} \\
\hline & & Stir-baking with rice wine & $0.044 \uparrow$ & \\
\hline & & None & 0.128 & \\
\hline \multirow[t]{2}{*}{12} & Berberine hydrochloride & Stir-baking with vinegar & $0.120 \downarrow$ & {$[13]$} \\
\hline & & Stir-baking with rice wine & $0.118 \downarrow$ & \\
\hline \multirow{3}{*}{13} & Columbamine & None & 0.262 & [13] \\
\hline & Columbamine & Stir-baking with vinegar & $0.068 \uparrow$ & \\
\hline & & None & 0.282 & \\
\hline \multirow[t]{2}{*}{14} & Palmatine hydrochloride & Stir-baking with vinegar & $0.120 \downarrow$ & {$[13]$} \\
\hline & & Stir-baking with vinegar & $1.177 \uparrow$ & \\
\hline \multirow{2}{*}{15} & I inosoluble comnonents & None & 0.932 & [9] \\
\hline & Liposoluble components & Stir-baking with vinegar & $0.711 \downarrow$ & [9] \\
\hline
\end{tabular}

PubChemCID: protopine: 4970; $\alpha$-allocryptopine: 98570; tetrahydrocolumbamine: 440229; coptisine: 72322; palmatine: 19009; berberine: 2353; dehydrocorydaline: 34781; tetrahydroberberine: 34458; corydaline: 10130; tetrahydrocoptisine: 6770; berberine hydrochloride: 12456; columbamine: 72310; palmatine hydrochloride: 73442. CAS registry number: D, L-tetrahydropalmatine: 10097-84-4 ( $\uparrow$ denotes that the content of corresponding compounds increased compared with the raw C. yanhusuo; $\downarrow$ denotes that the content of corresponding compounds decreased compared with the raw C. yanhusuo).

[20]. Despite the differences in experimental design, the results of the above studies showed that compounds in C. yanhusuo were easily absorbed and slowly eliminated. Only few reports have focused on the effects of pharmacokinetics characteristics on the therapeutic efficacy and toxicity after C. yanhusuo processing, and the relationship among the pharmacokinetics, therapeutic effect, and toxicity of processed C. yanhusuo remains unclear.

5.2. Distribution. Rat (Wistar species, $250 \pm 20 \mathrm{~g}$, male) was orally administered with processed C. yanhusuo extracts
$(10 \mathrm{~g} / \mathrm{kg})$ for 12 consecutive hours to compare the distribution of THP, dehydrocorydaline (DHC), and protopine in rat tissues. The ethanol extract of the unprocessed C. yanhusuo $(17.75 \mathrm{~g} / \mathrm{kg})$ and THP $(6.45 \mathrm{mg} / \mathrm{kg})$, DHC $(26.10 \mathrm{mg} / \mathrm{kg})$, and protopine $(4.52 \mathrm{mg} / \mathrm{kg})$ pure compound were used as the controls. The results showed that there were various effects on the $T_{\max }$ and MRT of DHC, THP, and protopine in processed C. yanhusuo, and the effect varies in different tissues and among different bioactive compounds indicating that differently processed C. yanhusuo products should be selected according to the specific disease condition and the affected organs [25]. 
However, the above study mainly focused on THP, DHC, and protopine consequently limiting comprehensive utilization and development of C. yanhusuo resources. Therefore, studies on the pharmacodynamics and pharmacokinetics of other alkaloids, such as protoberberine, isoquinoline, and nonalkaloids including steroids and organic acids, are required; this will lay a solid foundation for the follow-up research and development of new drugs.

5.3. Metabolism. In vivo studies showed that differentially processed $C$. yanhusuo formulations were metabolized differently. The elimination of compounds in C. yanhusuo in rats is a two-compartment model [26]. Rat (Wistar species, 200-250 g, male) was orally administered with processed C. yanhusuo extracts $(1.2 \mathrm{~g} / \mathrm{mL})$ for 24 consecutive hours to test the metabolism of THP, DHC, and protopine. The ethanol extract of the unprocessed C. yanhusuo $(1.2 \mathrm{~g} / \mathrm{kg})$ and THP $(0.516 \mathrm{mg} / \mathrm{kg})$, DHC $(0.361 \mathrm{mg} / \mathrm{kg})$, and protopine $(1.224 \mathrm{mg} / \mathrm{kg})$ pure compound were used as the controls. The results showed that there was significant raise in the elimination half-life of THP, protopine, and DHC compared with raw C. yanhusuo, and mean residence time was more than $6 \mathrm{~h}$, indicating that processing could delay the elimination of THP, protopine, and DHC [27, 28]. However, further studies on the specific mechanism of the synergistic effect are needed.

\section{Quality Control}

Tetrahydropalmatine is one of the alkaloids selected as a phytochemical marker for C. yanhusuo preparations quality control in the Chinese Pharmacopoeia. The Chinese Pharmacopoeia suggested that the quality of $C$. yanhusuo preparations should be based on THP properties, microscopic details, and its thin layer chromatography results. When evaluating C. yanhusuo quality using the hot dipping method together with the alcohol-soluble determination method, the amount of THP in C. yanhusuo should not be less than $0.04 \%$ (Chinese Pharmacopeia, 2020) [6]. However, using THP as the only quantitative marker may be insufficient in assessing the quality of $C$. yanhusuo, due to the impacts of other C. yanhusuo alkaloids on its physiological activity. Therefore, trahydroberberine and corydalis accounting for the analgesia, sedative, and hypnotic properties should also be considered for quality evaluation.

Different C. yanhusuo bioactive components, especially alkaloids, have been identified using highly sensitive technologies such as HPLC before and after processing [29]. For instance, the content of protopine, THP, CDL, berberine hydrochloride, and palmatine hydrochloride in C. yanhusuo was assessed using HPLC, and the average recovery was $100.12 \% \sim 100.98 \%$ (RSD $=1.05 \% \sim 1.90 \%, n=9$ ), suggesting that the HPLC method is an efficient quality control method for C. yanhusuo compounds [30]. Q-marker, a newly proposed concept of TCM quality control, recently used IP assays combined with chemical analysis, biosynthesis analysis, drug metabolism, and network pharmacology to identify the Q-markers of C. yanhusuo. Interestingly, the
Q-markers were found to be highly correlated with multiple C. yanhusuo biological activities, confirming the approach efficiency as a quality control method for C. yanhusuo before and after processing. However, there is still no suitable method for identifying Q-markers in C. yanhusuo complex compatibility system [31].

Dao-di herbs are medicinal herbs produced in specific areas, which have a long traditional use history and excellent medicinal efficacy [32]. An HPLC test on C. yanhusuo preparations from 15 production areas showed that the concentrations of CDL and THP were between $0.08-0.44 \%$ and $0.03-0.2 \%$, respectively, indicating that the alkaloid content in rhizomes differs significantly between samples from different habitats [33]. According to the "BencaoYuanshi” (《本草原始》) (Ming Dynasty, A.D. 1612), the best-quality medicinal $C$. yanhusuo is known as "Yuanhu" (Dao-di herbs), and it is found in Zhejiang Province. Nonetheless, further studies on the C. yanhusuo quality control evaluation are needed. Since no standard quality control methods and indicators are available, further studies incorporating a variety of alkaloids as potential candidates for C. yanhusuo quality evaluation index need to be conducted.

\section{Safety}

Based on previous clinical and animal trials, oral administration of raw C. yanhusuo extracts (more than 1-3 g/day) or total C. yanhusuo alkaloids $(473.36 \mathrm{mg} / \mathrm{kg})$ induced liver damage, muscle tremors, and renal hemorrhage in mice, indicating that raw C. yanhusuo extracts and total C. yanhusuo alkaloids have mild toxicity [34]. The liver damage may be due to the effects of high-dose C. yanhusuo including disordered energy metabolism and increased hepatocyte membrane permeability that facilitated the release of intracellular transaminase into the blood, eventually leading to liver cell swelling, acid change, necrosis, and inflammatory cell infiltration [35]. However, the mechanism through which C. yanhusuo induces muscle tremor and renal hemorrhage has not been reported, and therefore, further studies are needed.

The hepatotoxicity of total alkaloids in C. yanhusuo can be reduced by stir-baking with vinegar combined with Angelica sinensis and Curcumae longa. A GC-MS study found that hepatotoxicity was significantly decreased with the decreased content of total alkaloids in vinegar processed C. yanhusuo; this supported earlier reports that traditional processing could reduce toxicity [36]. Another experiment also confirmed that vinegar processing decreased the solubility of total C. yanhusuo alkaloids and reduced toxicity $[7,37]$. However, neither of the above studies identified the specific toxic components in C. yanhusuo. In addition, oral administration of polyherbal C. yanhusuo, Angelica sinensis, and Curcumae longa significantly alleviated mice muscle tremors and reduced hepatotoxicity [38]. However, the specific mechanism through which the combination reduced toxicity has not been clearly explained. According to the toxicity findings mentioned above, the toxicity of C. yanhusuo can be reduced by compatibility and dosage 
control. Meanwhile, oral administration of C. yanhusuo extracts (1-3 g/day) for 1-3 weeks is recommended as the optimal dosage for treatment of acute pain, cancer, and cardiovascular diseases [6]. C. yanhusuo is not recommended for patients with "Qi"-associated physical weakness with high body temperature, low levels of body fluids, acute abdominal pain, menorrhagia, and blood deficiency. Clinical studies have also reported that C. yanhusuo can increase the toxicity of Strychnos nux-vomica, which could be due to the formation of new toxic components as a result of combining C. yanhusuo and Strychnos nux-vomica. Therefore, C. yanhusuo and Strychnos nux-vomica should not be used concurrently [39]. In addition, after administration of THP pure compound, several cases were reported that THP possessed poison [40]. In this paper, we found that the concentration of THP in the tissues decreased rapidly after reaching the peak concentration and was only 1/17 1/49 of $C_{\max }$ after $12 \mathrm{~h}$, indicating that administration of C. yanhusuo instead of THP pure compound will not cause the accumulation of THP in the tissue [25].

In conclusion, raw C. yanhusuo extracts induce mild hepatotoxicity, mouse spasms, convulsions, and renal hemorrhage. Orally administered raw C. yanhusuo extracts and total alkaloids in C. yanhusuo should not exceed $3 \mathrm{~g} /$ day and $473.36 \mathrm{mg} / \mathrm{kg}$, respectively. Vinegar C. yanhusuo processing and combining C. yanhusuo with other medicinal plants are recommended approaches for reducing toxicity. However, C. yanhusuo toxic substances have been classified in a large category, namely, total alkaloids, and the specific toxic substances are still not clear, which requires further studies.

\section{Conclusions and Perspectives}

This review summarizes the phytochemistry, pharmacology, pharmacokinetics, quality control, and safety of C. yanhusuo under various processing and compatibility methods. Based on our findings, compared with raw C. yanhusuo, vinegar processing or polyherbal C. yanhusuo with Angelica dahurica showed increased analgesic effects, alleviated liver damage and mice muscle tremor caused by C. yanhusuo, and promoted the absorption of THP in the liver, suggesting that vinegar processing and combining C. yanhusuo with $A n$ gelica dahurica are the best processing and compatibility methods, respectively.

C. yanhusuo has been used for a long time in Asia as an analgesic, antitumor, anti-inflammatory, and cardiovascular protective agent. Several studies on rational processing, design, rational application mechanism of action, and toxicity of $C$. yanhusuo have so far been carried out. However, there are noticeable knowledge gaps that require further studies. First, there is no comprehensive research on synergism and attenuation effects of C. yanhusuo preparations; it is not clear whether new components are found after processing or compatibility, and information on the absorption, distribution, metabolism, and mechanism of action of new components is not available. Additionally, the mechanism of interaction between active components in C. yanhusuo compatibility preparations has not been clarified. Second, the effects of C. yanhusuo processing and compatibility on toxicity evaluation and toxicity mechanism remain unclear. Studies have shown decreased hepatotoxicity induced by C. yanhusuo with decreased content of total C. yanhusuo alkaloids in vinegar processed C. yanhusuo. However, the specific toxic alkaloid compounds and toxic metabolites are still unknown. In addition to the analgesic and sedative effects, C. yanhusuo has been reported to cause coronary artery dilation, lower blood pressure, and antiarrhythmia. The effects of processing and compatibility on these pharmacological effects need to be studied. Moreover, most studies are based on animal models, and therefore, clinical studies are needed to verify the therapeutic efficacy of processed C. yanhusuo. From the traditional view, processing increased the therapeutic effects of C. yanhusuo, while based on the findings, raw C. yanhusuo has been proved to have better antitumor activities than processed C. yanhusuo; this needs to be further investigated before clinical application. Finally, the vinegar and wine C. yanhusuo processing methods are the most commonly used and studied processing methods, which have limited the chances of exploring other processing methods. Due to several influencing factors, it is difficult to achieve unification with the processing technology, and consequent standardization of processing and quality control of TCM are also challenging. Beyond the alkaloids, more active compounds need to be examined during C. yanhusuo control quality, and quality standard of processed C. yanhusuo and its compatibility need to be established; this will help to ensure the optimal quality, efficacy, and safety of C. yanhusuo preparations.

\section{Conflicts of Interest}

The authors declare that they do not have any conflicts of interest.

\section{Authors' Contributions}

LW was responsible for collating documents and writing the manuscript; YY, DR, and ZM were responsible for the arrangement of tables and pictures; LQ and JW were responsible for providing important guidance on writing and revision of the review; $\mathrm{BZ}$ was responsible for analysis and manuscript preparation. All the authors significantly contributed to the article and approved the submitted version.

\section{Acknowledgments}

This study was supported by grants from the Zhejiang Provincial Natural Science Foundation of China (LQ21H280003 and LQ18H28009). The authors are grateful to Professor Qiaoyan Zhang for her careful proofreading and Mr. Kaohua Liu for his careful photographic work.

\section{Supplementary Materials}

Supplementary Table S1 provides examples of traditional Chinese medicine prescriptions containing Corydalis yanhusuo W.T.Wang. (Supplementary Materials) 


\section{References}

[1] B. C. Cai, Processing on Chinese Materia Medica, Chinese Medicine Press, Beijing, China, 2008.

[2] L. L. Chen, R. Verpoorte, H. R. Yen et al., "Effects of processing adjuvants on traditional Chinese herbs," Journal of Food and Drug Analysis, vol. 26, no. 2, pp. S96-s114, 2018.

[3] J. X. Wu, H. Zheng, X. Yao et al., "Comparative analysis of the compatibility effects of Danggui-Sini Decoction on a blood stasis syndrome rat model using untargeted metabolomics," Journal of Chromatography B, vol. 1105, pp. 164-175, 2019.

[4] B. Tian, M. Tian, and S. M. Huang, "Advances in phytochemical and modern pharmacological research of Rhizoma Corydalis," Pharmaceutical Biology, vol. 58, no. 1, pp. 265-275, 2020.

[5] Y. Zhou, X. Gao, C. Wu, and Y. Wu, "Bioaccessibility and safety assessment of trace elements from decoction of "Zhebawei" herbal medicines by in vitro digestion method," Journal of Trace Elements in Medicine \& Biology, vol. 28, no. 2, pp. 173-178, 2014.

[6] Chinese Pharmacopoeia, Pharmacopoeia Commission of Ministry of Health of the People's Republic of China, People's Medical Publishing House, Beijing, China, 2020.

[7] J. J. Liu, Y. L. Wei, Y. Z. Xin et al., "The modern research progress of the mechanism of enhancing efficacy and reducing toxicity of traditional Chinese medicine vinegar," Pharmaceutical Research, vol. 38, no. 8, pp. 474-476, 2019.

[8] M. Lefevre, P. M. Kris-Etherton, G. Zhao, and R. P. Tracy, "Dietary fatty acids, hemostasis, and cardiovascular disease risk11 continuing education questionnaire, page 492 meets learning need codes 4040, 4050, 5160, and 9020," Journal of the American Dietetic Association, vol. 104, no. 3, pp. 410-419, 2004.

[9] Y. X. Chen, F. Li, and Z. L. Zhou, "Analysis of liposoluble contents of no-processed and processed rhizoma Corydalis by GC-MS," Journal of Shandong University of Traditional Chinese Medicine, vol. 34, no. 3, pp. 251-253, 2010.

[10] Y. Luo, X. J. Ren, and H. Xu, "Research review on processing technology of Corydalis yanhusuo," Modern Agricultural Science and Technology, vol. 16, pp. 59-61, 2016.

[11] Z. Y. Zhou, W. R. Zhao, W. T. Shi et al., "Endothelial-dependent and independent vascular relaxation effect of tetrahydropalmatine on rat aorta," Frontiers in Pharmacology, vol. 10, no. 10, p. 336, 2019.

[12] H. Wu, K. Waldbauer, L. Tang et al., "Influence of vinegar and wine processing on the alkaloid content and composition of the traditional Chinese medicine Corydalis rhizoma (Yanhusuo)," Molecules, vol. 19, no. 8, pp. 11487-11504, 2014.

[13] H. W. Song, R. Mao, L. H. Li et al., "Study on the method of multi-component quality control before and after processing of Corydalis yanhusuo," Tianjin University of Traditional Chinese Medicine, vol. 38, no. 1, pp. 63-67, 2019.

[14] X. Wu, S. Wang, J. Lu et al., "Seeing the unseen of Chinese herbal medicine processing (Paozhi): advances in new perspectives," Chinese Medicine, vol. 13, no. 1, p. 4, 2018.

[15] S. L. Ingram, "Pain: novel analgesics from traditional Chinese medicines," Current Biology, vol. 24, no. 3, pp. R114-R116, 2014.

[16] Y. Y. Liu, T. X. Wang, J. C. Zhou, W. M. Qu, and Z. L. Huang, "Dopamine D1 and D2 receptors mediate analgesic and hypnotic effects of l-tetrahydropalmatine in a mouse neuropathic pain model," Psychopharmacology, vol. 236, no. 11, pp. 3169-3182, 2019.
[17] Y. L. Liu, L. D. Yan, P. L. Zhou, C. F. Wu, and Z. H. Gong, "Levo-tetrahydropalmatine attenuates oxycodone-induced conditioned place preference in rats," European Journal of Pharmacology, vol. 602, no. 2-3, pp. 321-327, 2009.

[18] Z. Qu, J. Zhang, L. Huo et al., "Antihypertensive and vasorelaxant effects of rhizoma Corydalis and its active component tetrahydropalmatine via NO/cGMP pathway and calcium channel blockade in isolated rat thoracic aorta," RSC Advances, vol. 5, no. 114, pp. 94130-94143, 2015.

[19] Z. C. Qiu, Y. X. Chen, and R. L. Zhou, "Comparative study between Rhizoma Corydalis processing with vinegar and clean Rhizoma Corydalis in anti-inflammatory effect and analgesic effect," Progress in Modern Biomedicine, vol. 9, p. 23, 2009.

[20] Z. G. Liao, X. L. Liang, J. Y. Zhu et al., "Correlation between synergistic action of Radix Angelica dahurica extracts on analgesic effects of Corydalis alkaloid and plasma concentration of dl-THP," Journal of Ethnopharmacology, vol. 4, no. 1, pp. 115-120, 2010.

[21] X. Zhao, J. Li, Y. Meng, M. Cao, and J. Wang, "Treatment effects of Jinlingzi powder and its extractive components on gastric ulcer induced by acetic acid in rats," Evidence-based Complementary and Alternative Medicine, vol. 2019, Article ID 7365841, 12 pages, 2019.

[22] J. L. Gao, T. C. He, Y. B. Li, and Y. T. Wang, "A traditional Chinese medicine formulation consisting of Rhizoma Corydalis and Rhizoma Curcumae exerts synergistic anti-tumor activity," Oncology Reports, vol. 22, no. 5, pp. 1077-1083, 2009.

[23] B. Cui, J. Yang, Z. Wang et al., "An ultra-high performance liquid chromatography with tandem mass spectrometry method for determination of 10 alkaloids in beagle dog plasma after the oral administration of the Corydalis yanhusuo W.T. Wang extract and Yuanhuzhitong tablets," Molecules, vol. 23, no. 8, p. 1925, 2018.

[24] C. Yang, S. Wang, X. Guo, J. Sun, L. Liu, and L. Wu, "Simultaneous determination of seven anthraquinones in rat plasma by ultra high performance liquid chromatographytandem mass spectrometry and pharmacokinetic study after oral administration of Semen Cassiae extract," Journal of Ethnopharmacology, vol. 169, pp. 305-313, 2015.

[25] Z. Dou, K. Li, P. Wang, and L. Cao, "Effect of wine and vinegar processing of rhizoma Corydalis on the tissue distribution of tetrahydropalmatine, protopine and dehydrocorydaline in rats," Molecules, vol. 17, no. 1, pp. 951-970, 2012.

[26] H. Zhang, X. Wu, J. Xu et al., "The comparative pharmacokinetic study of Yuanhu Zhitong prescription based on five quality-markers," Phytomedicine, vol. 44, no. 44, pp. 148-154, 2018.

[27] P. Wang, Z. Y. Dou, and L. Cao, "Pharmacokinetics of processed products of Rhizoma Corydalis in rat plasma," Journal of Chinese Pharmaceutical Sciences, vol. 44, no. 13, pp. 1013-1018, 2009.

[28] P. Wang, T. L. Zhang, G. H. Yu et al., "Poly-pharmacokinetic strategy-delineated metabolic fate of bioactive compounds in a traditional Chinese medicine formula, Yuanhu Zhitong tablets, using parallel reaction monitoring mode," Phytomedicine, vol. 53, pp. 53-61, 2019.

[29] Y. Lu, Q. Ma, C. Fu, C. Chen, and D. Zhang, "Quality evaluation of Corydalis yanhusuo by high-performance liquid chromatography fingerprinting coupled with multicomponent quantitative analysis," Scientific Reports, vol. 10, no. 1, p. 4996, 2020.

[30] P. Sun, Z. Guo, W. Yang et al., "Study on the fingerprint of crude/vinegar-processed Corydalis yanhusuo decoction pieces and their dispensing granules and content determination of 
five alkaloids," China Pharmacy, vol. 31, no. 20, pp. 24622468, 2020.

[31] K. Li, J. Li, J. Su et al., "Identification of quality markers of Yuanhu Zhitong tablets based on integrative pharmacology and data mining," Phytomedicine, vol. 44, pp. 212-219, 2018.

[32] S. S. Chu and H. S. Peng, "The evolution and change of Corydalis yanhusuo cableway herbs," Journal of Traditional Chinese Medicine, vol. 33, no. 6, p. 21, 2015.

[33] L. Chen, Study on the National Suitability Division Based on the Quality of Corydalis Yanhusuo, Master Thesis of Zhejiang Chinese Medical University, Zhejiang, China, 2017.

[34] J. H. Byeon, J. H. Kil, Y. C. Ahn, and C. G. Son, "Systematic review of published data on herb induced liver injury," Journal of Ethnopharmacology, vol. 233, pp. 190-196, 2019.

[35] J. L. Wang and C. F. Zhou, "Research progress of Chinese herbal medicine and traditional Chinese medicine resulting in liver injury," China Journal of Chinese Materia Medica, vol. 36, pp. 3371-3374, 2011.

[36] Z. Guo, R. Cai, H. Su, and Y. Li, "Alkaloids in processed rhizoma Corydalis and crude Rhizoma Corydalis analyzed by GC/MS," Journal of Analytical Methods in Chemistry, vol. 2014, Article ID 281342, 6 pages, 2014.

[37] L. P. Cheng, X. Z. Gu, and S. J. Mao, "Acute toxicity of different types of vinegar on mice and comparison of analgesic effect of processed Corydalis yanhusuo," Chinese Journal of Experimental Traditional Medical Formulae, vol. 16, no. 2, pp. 71-72, 2010.

[38] S. C. Wang, M. Y. Liu, and Y. W. Hu, "Toxic effects of Angelica, Curcuma, rhizoma corydalis and their compatibility on mice," Lishizhen Medicine and Materia Medica Research, vol. 4, no. 4, pp. 211-213, 2004.

[39] J. Yan, X. Y. Zhen, Z. Q. Ling, and J. D. Liu, "Experimental study on the toxicity of compatibility of Rhizoma Corydalis yanhusuo and Strychnosnus-vomica," China Journal of Traditional Chinese Medicine and Pharmacology, vol. 35, no. 7, pp. 3690-3694, 2020.

[40] C. K. Lai and A. Y. W. Chan, "Tetrahydropalmatine poisoning: diagnoses of nine adult overdoses based on toxicology screens by HPLC with diode-array detection and gas chromatography-mass spectrometry," Clinical Chemistry, vol. 45, no. 2, pp. 229-236, 1999. 